\title{
Clinical significance of serum matrix metalloproteinase 9 and tissue inhibitor of metalloproteinase 1 in the first phase of burn trauma evolution
}

\author{
ADINA ELENA STANCIU ${ }^{1 *}$, ADINA ZAMFIR-CHIRU-ANTON ${ }^{2 *}$, MARCEL MARIAN STANCIU $^{3}$, \\ MIRELA GHERGHE ${ }^{4,5^{*}}$, RAZVAN HAINAROSIE ${ }^{6,7}$, \\ FLORENTINA LIGIA FURTUNESCU ${ }^{8}$ and DAN CRISTIAN GHEORGHE ${ }^{7,9}$
}

${ }^{1}$ Department of Carcinogenesis and Molecular Biology, 'Prof. Dr. Alexandru Trestioreanu' Institute of Oncology, 022328 Bucharest; ${ }^{2}$ ENT Department, 'Grigore Alexandrescu' Children's Emergency Hospital, 011743 Bucharest; ${ }^{3}$ Electrical Engineering Faculty, 'Politehnica' University, 060042, Bucharest; ${ }^{4}$ Department of Nuclear Medicine,

'Prof. Dr. Alexandru Trestioreanu' Institute of Oncology, 022328 Bucharest; ${ }^{5}$ Department of Radiology, 'Carol Davila' University of Medicine and Pharmacy, 050463 Bucharest; ${ }^{6}$ ENT Department,

'Prof. Dr. Dorin Hociota' Institute of Phonoaudiology and Functional ENT and Cervicofacial Surgery, 050751 Bucharest;

${ }^{7}$ ENT Department, ${ }^{8}$ Department of Complementary Sciences, 'Carol Davila' University of Medicine and Pharmacy, 050463 Bucharest; ${ }^{9}$ ENT Department, 'Maria Sklodowska Curie’ Children's Emergency Hospital, 077120 Bucharest, Romania

Received April 29, 2021; Accepted June 3, 2021

DOI: $10.3892 /$ etm.2021.10547

\begin{abstract}
No prospective study has specifically examined the serum levels of matrix metalloproteinase-9 (MMP-9) and tissue inhibitor of metalloproteinase-1 (TIMP-1) in the early shock phase of burn-injured patients. Thus, we aimed to detect early changes, activity dynamics, and the predictive value of MMP-9, TIMP-1, and the MMP-9/TIMP-1 ratio to better understand the early repair mechanisms for the development of future therapies for patients with thermal burns. Twenty-five patients with a total body surface area (TBSA) affected by burn $<25 \%$, and 30 healthy subjects were enrolled in the study. Serum levels of MMP-9 and TIMP-1 were determined by the
\end{abstract}

Correspondence to: Dr Florentina Ligia Furtunescu, Department of Complementary Sciences, 'Carol Davila' University of Medicine and Pharmacy, 37 Dionisie Lupu Street, 050463 Bucharest, Romania E-mail: florentina.furtunescu@umfcd.ro

Dr Adina Elena Stanciu, Department of Carcinogenesis and Molecular Biology, 'Prof. Dr. Alexandru Trestioreanu' Institute of Oncology, 252 Fundeni Street, 022328 Bucharest, Romania

E-mail: adinaelenastanciu@yahoo.com

*Contributed equally

Abbreviations: MMP-9, matrix metalloproteinase-9; TIMP-1, tissue inhibitor of metalloproteinase-1; TBSA, total body surface area; ROC, receiver operating characteristic; AUC, area under the ROC curve

Key words: burn injury, MMP-9, TIMP-1, MMP-9/TIMP-1 ratio, ROC curve
ELISA method. Our results showed that MMP-9 concentrations increased immediately after injury and remained on a plateau. In contrast, TIMP-1 showed an upward trend throughout the 7-day study period, and the time course of the MMP-9/TIMP-1 ratio followed the inverse dynamics of TIMP-1. Analysis of the areas under the receiver operating characteristic (ROC) curves (AUC) showed that patients with burn wounds tended to have a MMP-9 value higher than $421.5 \mathrm{ng} / \mathrm{ml}$ (AUC=0.979), TIMP-1 value higher than $231.6 \mathrm{ng} / \mathrm{ml}(\mathrm{AUC}=0.908)$, and MMP-9/TIMP-1 ratio higher than 2.31 ( $\mathrm{AUC}=0.959)(\mathrm{P}<0.001)$. Our findings suggest that although the variations in the two biomarkers were different regarding the time of the initial insult, their ratio is a specific and sensitive indicator of burn evolutivity in patients with a TBSA affected by a burn $<25 \%$.

\section{Introduction}

After a severe burn injury, the wound healing response involves the dynamic interaction of many pathophysiological processes such as inflammation, proliferation, and tissue remodeling (1). The first phase consists of releasing a prolonged immune response (cytokines and chemokines in the endothelium) that activates proinflammatory effector cells at the site of injury and increases vasodilation and tissue edema (2). In early inflammation, increased levels of proinflammatory cytokines bring neutrophils and monocytes to the site (3). Further, proteolytic enzymes, such as matrix metalloproteinases (MMPs), are released from neutrophils (4). MMPs are the main class of enzymes responsible for the degradation or resorption of all extracellular matrix (ECM) components $(5,6)$.

Consequently, in acute inflammation, MMPs break down the ECM and basement membranes and contribute 
to vascular permeability in burn injury by stimulating remodeling of the connective tissue (7). The ideal tissue remodeling after injury requires a balance between MMPs and their tissue inhibitors (TIMPs), increasing TIMPs, resulting in either the accumulation of ECM or fibrosis. Simultaneously, the decrease in TIMPs leads to intense matrix proteolysis (5-8). Virtually, any imbalance between MMPs and TIMPs may lead to low healing processes such as chronic wounds or excessive healing, including hypertrophic scars and keloids (9).

Several studies have shown that matrix metalloproteinase-9 (MMP-9) and its tissue inhibitor (TIMP-1) are increased in plasma after thermal injury (10-12). MMP-9 is a dynamic marker, which is released from neutrophil granulocytes very early, according to the literature just 1-3 h after the triggering insult $(13,14)$. TIMP-1 is known to inhibit the catalytic activity of MMP-9 in a 1:1 stoichiometric relationship (15). Circulating levels of TIMP-1 are maximal two days after a severe burn (11). Unfortunately, the role of MMP-9 and TIMP-1 in early inflammation associated with a burn injury is poorly understood.

As no prospective study has specifically examined the serum levels of MMP-9 and TIMP-1 in the early shock phase of burn-injured patients, we aimed to detect early changes, activity dynamics, and predictive value of MMP-9, TIMP-1, and the MMP-9/TIMP-1 ratio to better understand the early repair mechanisms for the development of future therapies for patients with thermal burns.

\section{Patients and methods}

Patients and study protocol. This prospective study enrolled 25 patients with burn wounds (16 males/ 9 females, mean age $49.40 \pm 17.55$ years) admitted to the Clinical Emergency Hospital for Plastic, Reconstructive, and Burns Surgery in Bucharest, between 2018 and 2019. All cases had domestic accidents and among the reported comorbidities, high blood pressure was present in 4 patients. No history of diabetes or allergic reactions were reported.

The severity of burn trauma is represented by the burn depth and burn size. Burn size can be estimated by reference to the total body surface area (TBSA) using several methods [palms rule (16), nines rule (16), Lund and Browder chart (17), and mobile applications (17)]. Inclusion criteria included thermal burns with a TBSA affected by the burn $<25 \%$ and patients over 18 years of age. All burn patients were clinically assessed and presented with second- and third-degree burns. According to the updated guidelines for burn care (European Practice Guidelines for Burn Care), the treatment strategies for patients were carried out (18) and local protocols were followed. All patients were examined at admission and after 2 and 7 days. Exclusion criteria were as follows: i) patients of age under 18 years; ii) patients presenting with known cancer disease; iii) patients presenting with chronic heart failure or renal failure; iv) patients presenting with primary or secondary immunosuppressive disorders; v) patients receiving previous treatment with immunosuppressive drugs affecting the body's inflammatory response to burns (systemic corticosteroids); vi) patients receiving antibiotics from the tetracycline group, especially doxycycline (known as an MMP inhibitor) (19).
In addition, 30 healthy subjects (19 males/11 females, mean age $49.7 \pm 8.04$ years) were randomly selected and were similar in regards to the age and sex of the cases.

The study conformed to the principles outlined in the Declaration of Helsinki and was approved by the Clinical Emergency Hospital's Ethics Committee for Plastic, Reconstructive, and Burns Surgery. Written informed consent was obtained from enrolled patients or their legal representatives and volunteers.

Blood sample collection and processing. Blood samples were collected by venous puncture into BD Vacutainer ${ }^{\circledR} \mathrm{SST}^{\mathrm{TM}}$ Tubes (purchased from Becton, Dickinson and Company) as soon as possible after admission of the patients with burn wounds at the emergency department and following that at $48 \mathrm{~h}$ and 7 days. The serum samples were obtained by centrifugation at 2,500 x g for $15 \mathrm{~min}$ after $30 \mathrm{~min}$ of clotting time at room temperature. Serum samples were then immediately aliquoted into labelled cryo-vials and stored at $-70^{\circ} \mathrm{C}$ until further analysis.

Detection of serum MMP-9 and TIMP-1 by ELISA. The quantitative determination of serum MMP-9 and TIMP-1 concentrations was performed using ELISA kits purchased from R\&D Systems Inc. for human MMP-9 (cat. no. DMP900) and TIMP-1 (cat. no. DTM100), according to the manufacturer's instructions. Contamination may lead to falsely high serum concentrations. As MMP-9 and TIMP-1 are present in saliva, protective measures were taken to prevent contamination during the test. For both MMP-9 and TIMP-1, three serum samples of known concentration were tested 10 times on one plate to assess intra-assay precision and in 20 separate assays to assess inter-assay precision. The intra-assay precision values were identical to those of the inter-assay study with coefficients of variation (CVs) ranging from 2 to $7.9 \%$. The within CVs for MMP-9 were $1.9 \%$ at a mean concentration of $204 \mathrm{ng} / \mathrm{ml}$ and for TIMP-1 were $3.9 \%$ at a mean concentration of $127 \mathrm{ng} / \mathrm{ml}$. The MMP-9/TIMP-1 ratio was calculated using the following formula: MMP-9 (ng/ml)/TIMP-1 (ng/ml). All assays were performed in duplicate and in such a way, this minimized any effects of repeated freeze-thaw cycles.

Statistical analysis. Statistical analysis was conducted by using SPSS version 25 software (IBM Corp.). The difference between MMP-9 and TIMP-1 serum concentrations measured in dynamics was analyzed by one-way ANOVA test, comparing their evolution during the 7-day monitoring period from the initial burn injury. The Anderson-Darling, Shapiro-Wilk, and Kolmogorov-Smirnov tests were also used to verify the data obtained after preliminary analysis and to check the group's consistency. The diagnostic power of the MMP-9 and TIMP-1 biomarkers was assessed by calculating the areas under the receiver operating characteristic (ROC) curves (AUC). The ROC curves were plotted using as a variable the value of MMP-9, TIMP-1, or MMP-9/TIMP-1 ratio for patients admitted to the emergency department compared to the healthy controls, and as a classification criterion, the TBSA burn $<25 \%$. The AUC is an overall summary of diagnostic accuracy as follows: AUC >0.9, excellent diagnostic accuracy; AUC between 0.7 and 0.9, good diagnostic accuracy; AUC 

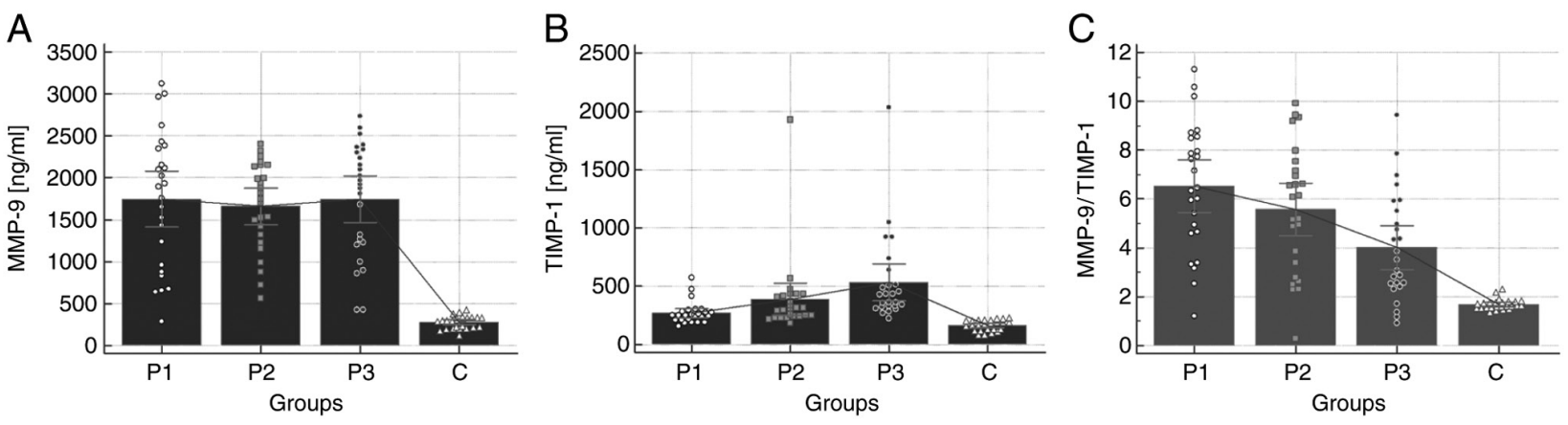

Figure 1. Dynamics of (A) MMP-9 serum concentrations, (B) TIMP-1 serum concentrations, and (C) MMP-9/TIMP-1 ratios during the study period in patients with skin burn injuries (P1, upon admission; P2, after 2 days; P3, after 7 days) in comparison to the control group. C, control group; MMP-9, matrix metalloproteinase-9; TIMP-1, tissue inhibitor of metalloproteinase-1.

between 0.5 and 0.7, poor diagnostic accuracy; $\mathrm{AUC}<0.5$, lack of diagnostic value of the biomarker (20). ROC curves have determined cut-off values for optimal sensitivity and specificity. For all tests, the significance level for statistical analysis was set at $\mathrm{P}$-values $<0.05$.

\section{Results}

Dynamics of MMP-9, TIMP-1, and MMP-9/TIMP-1 ratio. Fig. 1 depicts the dynamics of MMP-9, TIMP-1, and the MMP-9/TIMP-1 ratio in patients with burn injury compared to the healthy control group.

Serum MMP-9 concentrations upon admission (P1) and on days 2 (P2) and 7 (P3) were significantly higher in the burn patients than that in the healthy control group $(\mathrm{C})(\mathrm{P} 1$, $1,744.19 \mathrm{ng} / \mathrm{ml}$; P2, 1,659.62 ng/ml; P3, 1,744.95 ml vs. C, $279.36 \mathrm{ng} / \mathrm{ml}, \mathrm{P}<0.001)$. Basically, MMP-9 levels were maintained at a plateau throughout the trial period of 7 days (Fig. 1A).

TIMP-1 showed an increasing tendency during the entire study period (P1, $269.92 \mathrm{ng} / \mathrm{ml}$; P2, $385.86 \mathrm{ng} / \mathrm{ml}$; P3, $534.71 \mathrm{ng} / \mathrm{ml}$ vs. $\mathrm{C}, 166.68 \mathrm{ng} / \mathrm{ml}, \mathrm{P}=0.023)$, its levels being significantly higher on days 2 to 7 compared to admission levels (Fig. 1B).

The time course of the MMP-9/TIMP-1 ratio followed the inverse dynamics of TIMP-1. MMP-9/TIMP-1 ratios were significantly higher in the burn patients at all time points of the study (regardless of harvest time) compared to the healthy controls (P1, 6.51; P2, 5.58; P3, 4.008 vs. C, 1.68, P<0.001), following a continuous decrease from the day of admission until the end of the 7-day study interval (Fig. 1C).

Analysis of the ROC curves for MMP-9, TIMP-1, and $M M P-9 / T I M P-1$ ratio. The second objective of the present study was to compare the predictive value of MMP-9 with TIMP-1 and the MMP-9/TIMP-1 ratio in patients with burn injuries. The analysis of the ROC curves was performed to test the predictive value of the three biomarkers. AUC usually ranges from 0.5 to 1 , with values close to 1 indicating a high discriminatory ability. As shown in Fig. 2, all biomarkers had a very high AUC of over 0.90; the P-value being statistically significant in all analyzed cases.

The largest AUC was for MMP-9 (0.979); MMP-9 demonstrating an excellent accuracy for patients with TBSA affected by the burn $<25 \%$ (Fig. 2A). The ROC curve showed that these patients tended to have an MMP-9 value higher than $421.5 \mathrm{ng} / \mathrm{ml}$. This cut-off point was calculated for a sensitivity of $96 \%$ and a specificity of $100 \%$.

Analysis of the ROC curve for the TIMP-1 biomarker showed that patients with TBSA affected by the burn $<25 \%$ tended to have a TIMP-1 value greater than $231.6 \mathrm{ng} / \mathrm{ml}$; the cut-off point calculated for a sensitivity of $68 \%$ and a specificity of $100 \%$ (Fig. 2B). The AUC for TIMP-1 was very large (0.908) $(\mathrm{P}<0.001)$.

Considering the MMP-9/TIMP-1 ratio, AUC was extremely large $(0.959)(\mathrm{P}<0.001)$. Patients with a TBSA affected by the burn $<25 \%$ tended to have an MMP-9/TIMP-1 ratio greater than 2.31; the cut-off point calculated for a sensitivity of $96 \%$ and a specificity of $96.67 \%$ (Fig. 2C).

\section{Discussion}

The main findings of the current study were the following: i) MMP-9 serum levels were increased immediately after injury and remained at a plateau, while TIMP-1 showed an upward trend throughout the study period and the time course of MMP-9/TIMP-1 followed the inverse dynamics of TIMP-1; ii) the relationship between the MMP-9-TIMP-1 system and the extent of the injury was highly statistically significant $(\mathrm{P}<0.001)$; iii) analysis of the ROC curves showed that patients with a TBSA affected by the burn $<5 \%$ tended to have an MMP-9 value higher than $421.5 \mathrm{ng} / \mathrm{ml}(\mathrm{AUC}=0.979)$, a TIMP-1 value higher than $231.6 \mathrm{ng} / \mathrm{ml}(\mathrm{AUC}=0.908)$, and an MMP-9/TIMP-1 ratio higher than 2.31 (AUC=0.959).

The most common complications after burns are sepsis and respiratory, cardiac, and kidney failure, with the leading causes of death being heart failure $(67 \%)$ and respiratory failure (33\%) (13). None of the patients enrolled in the study had septic complications, but our case series included patients in a good health state and without a history of major non-communicable diseases, such as diabetes, which could influence the evolution of the burns (21). Although most studies have investigated MMP-9 or TIMP-1 immunohistochemical reactivity $(4,22)$, we decided to measure the serum level of biomarkers. Our decision was based on the following arguments: i) MMP-9, or TIMP-1 immunohistochemical reactivity may not correspond to in vivo enzymatic activity (12); ii) the study group was compared with the control group of healthy volunteers, 

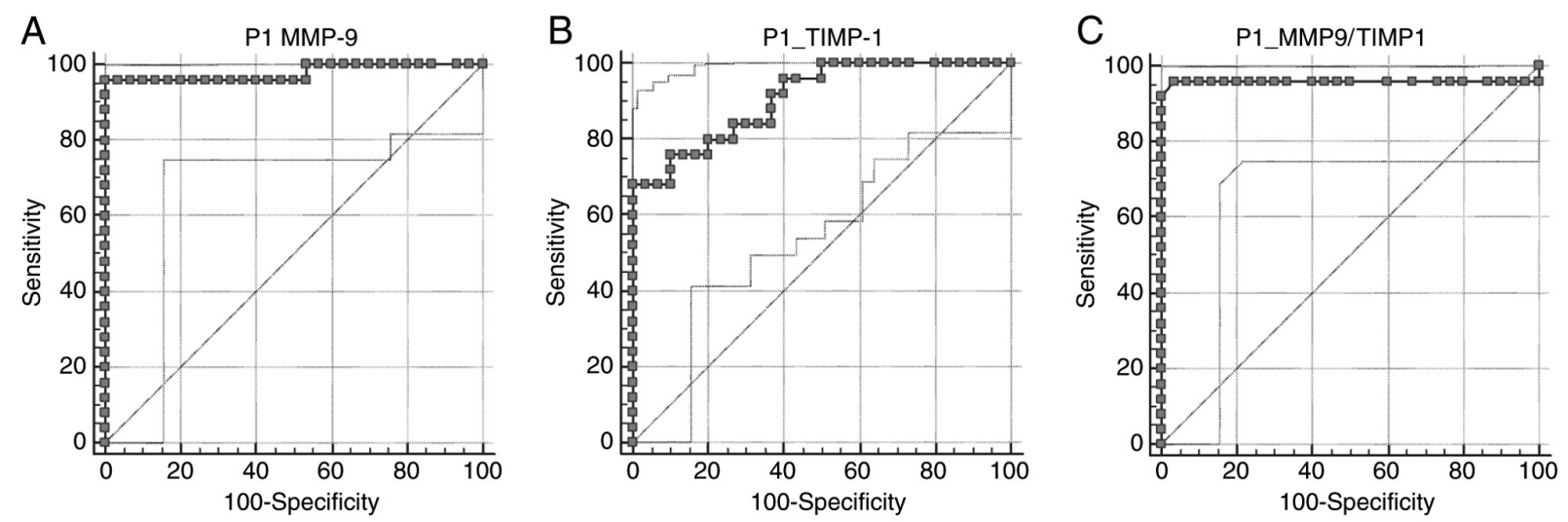

Figure 2. The receiver operating characteristic (ROC) curves for discriminating patients with a TBSA affected by the burn $<25 \%$ from healthy controls based on (A) MMP-9 serum concentrations, (B) TIMP-1 serum concentrations, and (C) MMP-9/TIMP-1 ratios. P1, upon admission; MMP-9, matrix metalloproteinase-9; TIMP-1, tissue inhibitor of metalloproteinase-1; TBSA, total body surface area.

without thermal injury. Moreover, the MMP-9 and TIMP-1 biomarkers were analyzed concerning the clinical variables and the outcome at $48 \mathrm{~h}$ and 7 days after injury. We hypothesized that the 7-day study interval opens a wide window of time that may be sufficient to detect both ascending and descending inflammatory responses; the 7-day period being the time interval in which the systemic inflammatory response syndrome post-injury usually occurs (14).

Starting with the idea that MMP-9 and TIMP-1 are dynamic biomarkers released very early after the triggering insult, we considered it useful to measure the changes in their serum concentrations, depending on the evolution of inflammation for one week. Dynamic changes in circulating levels of MMP-9 and TIMP-1 demonstrate their involvement in the early response after thermal injury. A moderate increase in MMP-9 serum concentration is beneficial because the epithelialization rate is partially dependent on the presence of collagen. However, when the physiological balance between protease and their inhibitors is broken, the patient outcome is unfavorable (9). Our results showed an early increase in the MMP-9 level, most likely due to its rapid release from neutrophil granulocytes, over minutes to a few hours after the triggering insult (P1, $1744.19 \mathrm{ng} / \mathrm{ml}$ vs. C, $279.36 \mathrm{ng} / \mathrm{ml}, \mathrm{P}<0.001)$. Other studies described a significantly lower level of MMP-9 on days 4 to $6(14,23)$. In contrast to these studies, in the present study, MMP-9 levels were maintained at a plateau throughout the trial period of 7 days, which may lead us to the hypothesis that an early increased expression of MMP-9 (cut-off, $412.5 \mathrm{ng} / \mathrm{ml}$ ) could be associated with a better-quality scar and the maintenance/decrease in MMP-9 serum concentrations may be an indicator for better survival.

Several authors found significantly higher TIMP-1 concentrations in the plasma of TBSA $<20 \%$ burn patients relative to healthy controls, with a median time to peak TIMP-1 concentration at 2.09 days $(10,11,14)$. In contrast to these studies, our results showed that TIMP-1 presented an increasing tendency during the entire study period, its levels being significantly higher on days 2 to 7 compared to admission levels. According to the findings of Ulrich et al (11), TIMP-1 serum expression has been correlated with the TBSA\% of injury; patients with TBSA affected by the burn $<25 \%$ tending to have a TIMP-1 value greater than $231.6 \mathrm{ng} / \mathrm{ml}$. The high response of TIMP-1 to burns could explain the lower serum levels of MMP-9 than expected in our study.

As shown in Fig. 1, at all-time points of the study, the differences between serum concentrations of MMP-9 and its inhibitor TIMP-1 in the study group compared to the control group were statistically significant, with the time-course of the MMP-9/TIMP-1 ratio following the inverse dynamics of TIMP-1. The constant decrease in the MMP-9/TIMP-1 ratio throughout the trial period of 7 days (decrease in $14.28 \%$ at 2 days and decrease in $38.43 \%$ at 7 days from the value measured from admission) indicates that the patients with a TBSA affected by the burn $<25 \%$ have an effective healing process without hypertrophic scars and keloids.

Monitoring the levels of MMP-9 was not the most accurate indicator of the time elapsed from the initial injury. TIMP-1 proved to be better in this respect, and the MMP-9/TIMP-1 ratio showed the best sensitivity and specificity.

Healing processes evolve, as clinical practice demonstrates, after necrotic tissues fall off. The factors influencing local changes are not entirely known, but we can hypothesize that local proteinases can be responsible for tissue remodeling. In this respect, MMP-9 or TIMP-1 monitoring could prove to be useful markers for local changes over time, indicating the need for eventual surgery or other therapeutic approaches.

The main limitation of our work is related to the small number of patients included. However, despite the small sample size, we used a homogenous population because none of the patients enrolled in our study had septic complications.

In summary, our study found a significant increase in serum levels of MMP-9 and TIMP-1 in patients with a TBSA affected by the burn $<25 \%$, compared to unaffected controls. Although the variations in the two biomarkers were different regarding the time of the initial insult, their ratio is a specific and sensitive indicator of burn evolutivity. Further investigations comparing local and general protease responses after such trauma may provide new insights into the mechanism involved in burn wound healing. Detailed studies are needed to elucidate the exact sequence of local events that occur in the first 7 days after severe burn injury to better predict when local inflammation triggers systemic inflammatory processes. 


\section{Acknowledgements}

Not applicable.

\section{Funding}

This research did not receive any specific grant from any funding agency in the public, commercial or not-for-profit sector.

\section{Availability of data and materials}

All data generated or analyzed during this study are included in the manuscript.

\section{Authors' contributions}

AES and AZCA conceived and planned the experiments. Experiments were performed by AES. AZCA, FLF, and MMS performed statistical analysis of the results. AES, AZCA, MMS, MG, RH, FLF and DCG contributed to the interpretation of the results. AES took the lead in writing the manuscript. All authors provided critical feedback and helped shape the research, analysis, and manuscript. All authors read and approved the final manuscript for publication.

\section{Ethics approval and consent to participate}

The study conformed to the principles outlined in the Declaration of Helsinki and was approved by the Clinical Emergency Hospital for Plastic, Reconstructive, and Burns Surgery Ethics Committee (approval no. 6627/04.10.2017). Written informed consent was obtained from the enrolled patients or their legal representatives and volunteers.

\section{Patient consent for publication}

Not applicable.

\section{Authors' information}

Adina Elena Stanciu: ORCID ID: https://orcid.org/00000002-9494-6686.

\section{Competing interests}

The authors declare that they have no competing interests.

\section{References}

1. Lateef Z, Stuart G, Jones N, Mercer A, Fleming S and Wise L: The cutaneous inflammatory response to thermal burn injury in a murine model. Int J Mol Sci 20: 538, 2019.

2. Hofmann E, Fink J, Eberl A, Prugger EM, Kolb D, Luze H, Schwingenschuh S, Birngruber T, Magnes C, Mautner SI, et al: A novel human ex vivo skin model to study early local responses to burn injuries. Sci Rep 11: 364, 2021.

3. Suceveanu AI, Mazilu L, Katsiki N, Parepa I, Voinea F, Pantea-Stoian A, Rizzo M, Botea F, Herlea V, Serban D and Suceveanu AP: NLRP3 inflammasome biomarker-could be the new tool for improved cardiometabolic syndrome outcome. Metabolites 10: 448, 2020.
4. Yu G, Li Y, Ye L, Wang X, Zhang J, Dong Z and Jiang D: Exogenous peripheral blood mononuclear cells affect the healing process of deep-degree burns. Mol Med Rep 16: 8110-8122, 2017.

5. Cabral-Pacheco GA, Garza-Veloz I, Castruita-De la Rosa C, Ramirez-Acuña JM, Perez-Romero BA, Guerrero-Rodriguez JF, Martinez-Avila N and Martinez-Fierro ML: The roles of matrix metalloproteinases and their inhibitors in human diseases. Int J Mol Sci 21: 9739, 2020.

6. Raeeszadeh-Sarmazdeh M, Do LD and Hritz BG: Metalloproteinases and their inhibitors: Potential for the development of new therapeutics. Cells 9: 1313, 2020.

7. Plichta JK, Holmes CJ, Gamelli RL and Radek KA: Local burn injury promotes defects in the epidermal lipid and antimicrobial peptide barriers in human autograft skin and burn margin: Implications for burn wound healing and graft survival. J Burn Care Res 38: e212-e226, 2017.

8. Tokuhara CK, Santesso MR, Oliveira GSN, Ventura TMDS, Doyama JT, Zambuzzi WF and Oliveira RC: Updating the role of matrix metalloproteinases in mineralized tissue and related diseases. J Appl Oral Sci 27: e20180596, 2019.

9. Guo HF, Ali RM, Hamid RA, Chang SK, Rahman MH, Zainal Z and Khaza'ai $\mathrm{H}$ : Temporal changes in the cell population and wound healing-related gene expression in deep partial-thickness burn wound model. Biomed Dermatol 4, 2020.

10. Dasu MR, Spies M, Barrow RE and Herndon DN: Matrix metalloproteinases and their tissue inhibitors in severely burned children. Wound Repair Regen 11: 177-180, 2003.

11. Ulrich D, Noah EM, von Heimburg D and Pallua N: TIMP-1, MMP-2, MMP-9, and PIINP as serum markers for skin fibrosis in patients following severe burn trauma. Plast Reconstr Surg 111: 1423-1431, 2003.

12. Hästbacka J, Fredén F, Hult M, Bergquist M, Wilkman E, Vuola J, Sorsa T, Tervahartiala T and Huss F: Matrix Metalloproteinases- 8 and -9 and tissue inhibitor of metalloproteinase- 1 in burn patients A prospective observational study. PLoS One 10: e0125918, 2015.

13. Lang TC, Zhao R, Kim A, Wijewardena A, Vandervord J, Xue M and Jackson CJ: A critical update of the assessment and acute management of patients with severe burns. Adv Wound Care (New Rochelle) 8: 607-633, 2019.

14. Nagy B, Szelig L, Rendeki S, Loibl C, Rézmán B, Lantos J, Bogár L and Csontos C: Dynamic changes of matrix metalloproteinase 9 and tissue inhibitor of metalloproteinase 1 after burn injury. J Crit Care 30: 162-166, 2015.

15. Stanciu AE, Zamfir-Chiru-Anton A, Stanciu MM, Pantea-Stoian A, Nitipir C and Gheorghe DC: Serum melatonin is inversely associated with matrix metalloproteinase-9 in oral squamous cell carcinoma. Oncol Lett 19: 3011-3020, 2020.

16. Giretzlehner M, Dirnberger J, Owen R, Haller HL, Lumenta DB and Kamolz LP: The determination of total burn surface area: How much difference? Burns 39: 1107-1113, 2013.

17. Chong HP, Quinn L, Jeeves A, Cooksey R, Lodge M, Carney B and Molony D: A comparison study of methods for estimation of a burn surface area: Lund and Browder, e-burn and Mersey Burns. Burns 46: 483-489, 2020.

18. Brychta P, Magnette A, and the Executive Committee and PAM Committee of European Burn Association (EBA) (2017). European Practice Guidelines for Burn Care 2017. Available from: https://www.euroburn. org/wp-content/uploads/EBA-Guidelines-Version-4-2017.pdf.

19. Stagg HW, Whaley JG, Tharakan B, Hunter FA, Jupiter D, Little DC, Davis ML, Smythe WR and Childs EW: Doxycycline attenuates burn induced microvascular hyperpermeability. J Trauma Acute Care Surg 75: 1040-1046, 2013.

20. Stanciu AE, Zamfir-Chiru-Anton A, Stanciu MM, Stoian AP, Jinga V, Nitipir C, Bucur A, Pituru TS, Arsene AL, Dragoi CM, et al: Clinical significance of serum melatonin in predicting the severity of oral squamous cell carcinoma. Oncol Lett 19: 1537-1543, 2020

21. Stoian Pantea A, Mitrofan G and Colceag F: Oxidative Stress in Diabetes. A model of complex thinking applied in medicine. Rev Chim 96: 2515-2519, 2018.

22. Stanciu AE: Cytokines in heart failure. Adv Clin Chem 93: 63-113, 2019

23. Lorente L,Martin MM,Labarta L,DiazC,Solé-ViolánJ,Blanquer J, Orbe J, Rodriguez JA, Jimenez A, Borreguero-Leon JM, et al: Matrix metalloproteinase-9, -10, and tissue inhibitor of matrix metalloproteinases-1 blood levels as biomarkers of severity and mortality in sepsis. Crit Care 13: R158, 2009. 